
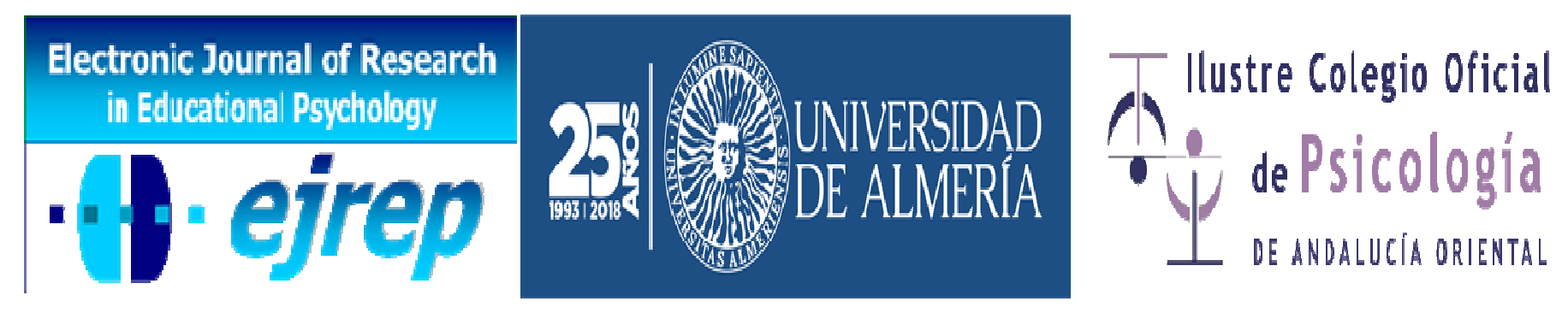

\title{
La relevancia de la formación y el contacto previo en la opinión de estudiantes universitarios sobre las personas con trastorno mental
}

\section{Domingo Barroso-Hurtado ${ }^{1}$, Santiago Mendo-Lázaro ${ }^{2}$}

\author{
${ }^{1}$ Departamento de Pedagogía, Universidad de Granada, Granada \\ ${ }^{2}$ Departamento de Psicología y Antropología, Universidad de Extremadura, Cá- \\ ceres
}

\section{España}

Correspondencia: Domingo Barroso-Hurtado, Departamento de Pedagogía, Facultad de Ciencias de la Educación, Universidad de Granada. Campus Universitario de la Cartuja s/n, 18071, Granada (España).

E-mail: dobarroso@ugr.es

(C) Universidad de Almería and Ilustre Colegio Oficial de la Psicología de Andalucía Oriental (Spain) 


\section{Resumen}

Introducción. En la presente investigación, se han analizado las diferencias en la opinión de los estudiantes universitarios hacia las personas con trastorno mental, en función de estos si han mantenido un contacto previo con las mismas y si han recibido formación en relación a ellas.

Método. Se aplicó la Escala de Opiniones sobre la Enfermedad Mental en Población Española (OMI-E) a una muestra de 474 estudiantes de la Universidad de Extremadura (España), a los que se preguntó si habían mantenido dicho contacto y de los cuales 279 cursaban el Grado en Educación Social

Resultados. La formación recibida parece ejercer una mayor influencia que el contacto previo Además, dicho contacto parece producir diferencias significativas únicamente en aquellos estudiantes que han recibido esa formación.

Discusión y conclusiones. Se desprende la conveniencia de realizar acciones de educación basada en el contacto, ya que pueden favorecer que este último tenga un carácter positivo y significativo.

Palabras Clave: trastorno mental, opinión, estudiantes universitarios, formación, contacto previo. 


\begin{abstract}
Introduction. In the present research, the differences in the view of the university students toward people with mental disorder have been analyzed, depending on whether they have had a previous contact with these people and if they have received training on them.

Method. The Opinion about mental illness scale adapted to Spanish population (OMI-E) was administered to a sample of 474 students of the University of Extremadura, who were also asked about if they had had such contact, and of whom 279 were studying the Degree in Social Education-
\end{abstract}

Results. Training seems to produce a greater influence than previous contact. Moveover, such contact seems to produce significant differences only in those subjects who have received such training and not on those who did not.

Discussion and Conclusion. From these results, performing actions of contact-based education, which probably favor that contact produces positive and significant effects, is recommended.

Keywords: mental disorders, opinions, stigmatization, college students, experience, training, previous contact. 


\section{Introducción}

El estigma se entiende como una marca que recae en forma de atributo profundamente desacreditador sobre un colectivo (Goffman, 2006). En el caso de aquél formado por las personas con trastorno mental, éste se podría traducir en creencias, actitudes, respuestas emocionales y comportamientos que pueden trascender en consecuencias adversas para los individuos que lo componen (Brown, 2012). Entre dichas consecuencias, se encuentran la presencia de limitaciones para su participación en la sociedad y en el acceso a los servicios que en ésta se prestan (Chen, Koller, Krupa y Stuart, 2016), un aumento de su ritmo de cronificación y de su exclusión (Lolich y Leiderman, 2008) o ser objeto de un mayor deseo de distancia social (Boyd, Katz, Link y Phelan, 2010), entendida como la intención de evitar el contacto o mantener una relación en diferentes niveles respecto a ellas (Fresán et al., 2012).

En los estudios sobre la reducción del estigma, a pesar de que el manual de diagnóstico DSM 5 recoge un total de 216 trastornos, es frecuente el uso generalizado del término "trastorno mental", el cual se utiliza como una categoría general que hace referencia a los diferentes tipos que existen (Delgado, 2015). Según dicho manual, se entiende por trastorno mental un síndrome derivado de una disfunción de los procesos subyacentes al funcionamiento mental -ya sean psicológicos, biológicos o del desarrollo-, que se relacionan con una alteración a nivel cognitivo, de comportamiento o de regulación emocional de un sujeto, la cual ha de ser significativa a nivel clínico y debe repercutir, a nivel de malestar o discapacidad, en actividades relevantes en la vida de un sujeto (American Psychological Association, 2013).

No obstante, los estigmas no afectan únicamente a las personas que componen este colectivo. También pueden hacerlo sobre su familia, círculo social cercano y los profesionales que trabajan con ellas (Lolich y Leiderman, 2008), los cuales, además de sufrirlos también pueden mantenerlos. En este sentido, determinadas investigaciones que apuntan a que estudiantes de Trabajo Social desean establecer cierta distancia social hacia las personas con estos trastornos (Covarrubias y Han, 2011) o que profesionales relacionados con el ámbito de la rehabilitación psicosocial presentan niveles de estereotipos hacia ellas, similares a la población general (Nordt, Rössler y Lauber, 2006). Por ello, conocer el impacto de diferentes estrategias dirigidas a la reducción del estigma en grupos de profesionales de dicho ámbito resulta de especial interés. 


\section{Estrategias para la reducción del estigma}

Se han articulado diferentes estrategias en un intento de reducir el impacto de los estereotipos, los prejuicios y, en última instancia, de la estigmatización sobre las personas con trastorno mental. Entre ellas, se encuentran la educación, consistente en un aumento del conocimiento y de la formación sobre estas personas, y el contacto social, centrado en la exposición o la interacción con las mismas (Delgado, 2015). No obstante, no existe consenso a la hora de establecer cuál de ellas resulta más adecuada. Mientras que por un lado, algunos autores destacan el papel del contacto social o la exposición a la hora de generar actitudes positivas y de reducir la estigmatización hacia las personas con trastorno mental (Álvarez y Pernía, 2007), por otro lado, algunos sostienen una mayor influencia de las estrategias basadas en la educación (Smith, 2008), las cuales pueden potenciar la construcción de imágenes más positivas y que se prefiera una menor distancia social respecto a estas personas (Lolich y Leiderman, 2008), así como una mejora en las actitudes hacia ellas (Álvarez y Pernía, 2007).

Esta falta de consenso también se extiende al impacto de dichas estrategias sobre la opinión y las actitudes que los estudiantes universitarios mantienen sobre las personas con trastorno mental. En cuanto a la educación, por una parte, la influencia de la formación sobre los estereotipos, las actitudes y los comportamientos estigmatizantes hacia estas personas ha sido probada en profesionales y estudiantes de diferentes ámbitos (Pingani et al., 2016; Sharp, Hargrove, Johnson y Deal, 2006; Smith, 2008). En cambio, otras investigaciones no han hallado relación entre la formación sobre trastornos mentales y la reducción del estigma (Covarrubias y Han 2011), ni un impacto significativo en la opinión y las actitudes de los estudiantes universitarios (Fresán et al., 2012; González, Tinsley y Kreuder, 2002; Zellmann, Madden y Aguiniga, 2014). En este sentido, por ejemplo, la educación podría no ser efectiva si se centra en explicar los trastornos mentales, principalmente en función de causas biogenéticas, ya que esto suele favorecer percepciones sobre las personas que los tienen como peligrosas, impredecibles, poco responsables de sus actos y, además, no suele favorecer la reducción de la estigmatización que pueden sufrir (Mannarini y Boffo, 2013).

Además, resultaría interesante atender a la influencia de las motivaciones previas que llevaron a estas personas a recibir dicha formación y a encontrarse cursando una u otra titulación, como es el caso del altruismo en titulaciones de corte más humanista como el Magisterio, la Medicina y la Psicología (Martínez, Castro, Lucena y Zurita, 2015). Por otro lado, la 
influencia del contacto social con personas que tienen un trastorno mental ha sido probada con estudiantes de diferentes ámbitos, entre los que se encuentra la salud mental (MedinaOrtíz et al., 2010; Smith, 2008), destacando tanto el contacto indirecto (Nguyen, Chen y O'Reilly, 2012), como el directo, regular y cercano (Brown, 2012). Sin embargo, otras investigaciones no hallaron una relación entre contacto y la reducción de las actitudes negativas (Fresán et al., 2012) o, incluso, encontraron un aumento de las mismas (Omori et al., 2012).

En las estrategias basadas en el contacto, es conveniente atender al tipo del mismo que se produce (ej.: directo o indirecto con diferentes grados de experiencia; Senra-Rivera, Arriba-Rossetto y Seoane-Pesqueira, 2008) o las condiciones en las que éste se da. En este sentido, para la reducción del prejuicio, puede resultar interesante que el contacto intergrupo se realice siguiendo las indicaciones de Allport (1958), como que se dé una igualdad de estatus y cooperación entre los grupos, aunque autores como Pettigrew y Tropp (2006) señalan que dichas indicaciones, aunque positivas, no son estrictamente necesarias. Además, en ese contacto entre grupos, los factores afectivos (empatía y reducción de la ansiedad) destacan sobre los cognitivos (conocimiento sobre el otro grupo) como mediadores en la reducción del prejuicio, aunque ambos ejercen una influencia positiva (Pettigrew y Tropp, 2008).

Por otra parte, confrontar los estereotipos sobre un grupo con información que no concuerde con sus contenidos, puede ser una de las vías más eficaces de cara a su modificación (Fernández, 2005). En referencia a los procesos cognitivos individuales de cambio, cabe destacar los modelos de contabilidad, conversión y subtipos que pueden desarrollarse cuando se presenta dicha información desconfirmatoria (Crocker y Weber, 1983).

Por último, como una combinación de las estrategias anteriores, se encontraría una educación basada en el contacto, la cual puede favorecer la adquisición de conocimientos, actitudes e intenciones del comportamiento más positivos hacia las personas con trastorno mental (Chen et al., 2016), así como puede resultar muy efectiva en la reducción del estigma (Delgado, 2015). En este sentido, determinadas investigaciones señalan que educación y contacto, por separado, no influyen en un cambio positivo en las actitudes hacia las personas con esquizofrenia y sugieren que ambas variables se necesitan entre sí para que éste se produzca. Por ello, exponen que para que un contacto social de carácter personal sea efectivo es necesario poseer conocimientos previos y que para que el conocimiento se asocie positivamente con 
ese cambio actitudinal es necesario que vaya acompañado de un contacto social (Eack y Newhill, 2008; Eack, Watson y Newhill, 2012).

\section{Objetivos}

Teniendo en cuenta todo lo anterior, en un intento de aportar información útil sobre las estrategias para la reducción del estigma hacia personas con trastorno mental, en la presente investigación se pretende analizar las diferencias en la opinión de los estudiantes universitarios hacia las personas con trastorno mental, en función de dos variables: la formación recibida sobre las personas con trastorno mental y la existencia de contacto previo con ellas.

\section{Método}

\section{Participantes}

Los participantes en este estudio han sido 474 estudiantes de la Universidad de Extremadura (España), de los cuales, el 22\% son varones y el $78 \%$ son mujeres. La edad media de la muestra es de 21.23 años $(D T=3.92)$. Del total de estudiantes, 275(58\%) han mantenido un contacto previo con personas con trastorno mental, 279 (59\%) cursan el Grado en Educación Social y 195 (41\%) estudian titulaciones pertenecientes a ramas de conocimientos que no encuentran a las personas con trastorno mental entre sus destinatarios principales (Filología Hispánica, Lenguas y Literaturas Modernas, Filología Clásica, Derecho, Administración y Dirección de Empresas). Dentro de su titulación, 201 (42,41\%) estudiantes están en el primer curso, $81(17,09 \%)$ en el segundo, $120(25,32 \%)$ en el tercero y $70(14,77 \%)$ en el cuarto.

A la hora de establecer los grupos de comparación, se ha dividido al alumnado en dos grupos. Por una parte, se encuentran los estudiantes de "Grado en Educación Social”, los cuales se han categorizado como sujetos con formación sobre el colectivo de personas con trastorno mental, al haber cursado materias relacionadas con este colectivo desde el comienzo de la carrera (y sobre otros temas como los estereotipos, los prejuicios y la rehabilitación) y porque, además, serán profesionales que podrán trabajar en el ámbito de la rehabilitación psicosocial (Universidad de Extremadura, 2016).

Por otra parte, se encuentran estudiantes que no han recibido formación específica sobre el colectivo en su plan de estudios, ya que sus profesiones no encuentran a las personas 
con trastorno mental entre sus destinatarios principales. Estos estudiantes se han recogido en la categoría de "otras titulaciones".

\section{Instrumentos}

Escala de Opiniones sobre la Enfermedad Mental en Población Española (OMI-E). Para la realización de esta investigación se ha empleado la traducción de la Escala de Opiniones sobre la Enfermedad Mental [Opinion about mental illness scale] (Cohen and Struening, 1962), realizada por Fresán et al. (2012). Al ser una escala originalmente aplicada en población mexicana, se han cambiado algunas palabras y/o expresiones por otras de uso más común en España (ej. "colonia" por "barrio" o "no se debería de hacer amistad con ellos" por "no se debería entablar amistad con ellos"). Asimismo, cabe destacar la sustitución del término enfermedad mental por trastorno mental, empleando de esta manera la terminología más aceptada por la comunidad científica y la cual está recogida en el DSM 5 de la American Psychological Association (2013).

Además, con el objetivo de analizar las relaciones entre la formación recibida y contacto previo con personas con trastorno mental, como factor adicional, de manera similar a investigaciones previas (Serrani, 2012), se incluyó un ítem relativo al mantenimiento de un contacto previo con personas con trastorno mental, sin establecer diferencias en función de los diversos tipos del mismo.

La OMI-E consta de 34 ítems que se miden a través de una escala tipo Likert con cinco opciones de respuesta, siendo $1=$ totalmente en desacuerdo y $5=$ totalmente de acuerdo. Los 34 ítems se agrupan en seis factores: 1) Separatismo (10 ítems), hace referencia a la distancia social que se desea mantener para estar alejado de las personas con trastorno mental, enfatizando las diferencias percibidas respecto a las mismas, ej. "Los pacientes psiquiátricos no deberían ser tratados en el mismo hospital en que se tratan a otros pacientes"; 2) Estereotipos (4 ítems), en referencia a la posesión de estos sobre el colectivo en cuestión, categorizándolo en un patrón de comportamiento y en una capacidad intelectual determinada, ej. "Todas las personas con un trastorno mental tienen un comportamiento extraño"; 3) Restricción (4 ítems), se refiere al mantenimiento de un punto de vista un tanto limitador en cuanto a los derechos de las personas con trastorno mental, ej. "Las personas con trastorno mental no deberían de tener hijos"; 4) Benevolencia (8 ítems), hace referencia a la compasión hacia este colectivo, ej. "La mejor forma de ayudar a las personas con trastornos mentales a recuperarse 
es integrarlos en la comunidad para tener una vida normal"; 5) Predicción pesimista (4 ítems), se refiere a la falta de expectativas en la mejoría de las personas con trastorno mental y percepción de que la sociedad tampoco se muestra optimista al respecto, ej. "Después del tratamiento es difícil para las personas con trastornos mentales regresar a la comunidad"; 6) Estigmatización (4 ítems), incluye ítems que se refieren a la percepción del trastorno mental como algo de lo que avergonzarse y que se debe de tratar de ocultar, ej. "Sugiero que aquéllos que tienen un trastorno mental no le digan a nadie acerca de su padecimiento".

El índice alpha de Cronbach muestra que el OMI-E presenta una adecuada fiabilidad global $(\alpha=.82)$, y aceptable de los seis factores de la escala [F1 $(\alpha=.81)$ F2 $(\alpha=.70)$; F3 ( $\alpha=$ $.71)$; F4 $(\alpha=.79)$; F6 $(\alpha=.68) ;$ F5 $(\alpha=.69)]$.

\section{Procedimiento}

Con una duración de 15 minutos, la Escala de Opiniones sobre la Enfermedad Mental (OMI-E), se aplicó al alumnado mientras se encontraba en el aula, previo consentimiento de los estudiantes y el profesorado responsable y tras haberse realizado una presentación sobre la finalidad de la aplicación de la escala y las instrucciones necesarias para cumplimentación. Además, se prestó especial atención a señalar que los datos obtenidos serían anónimos y tratados únicamente con fines de investigación, así como se solicitó a los participantes su consentimiento verbal sobre la utilización de la información proporcionada y su participación de forma voluntaria.

\section{Análisis de datos}

Previamente a la realización de los análisis estadísticos se somete a los datos a las pruebas de: Kolmogorov-Smirnov para analizar el supuesto de normalidad; Rachas para contrastar el supuesto de aleatorización, y Levene en las comparaciones de medias, contrastando así el supuesto de homoscedasticidad. Se encuentra $p>0,05$ en todos los contrastes, quedando justificada la utilización de pruebas paramétricas t de Student para muestras independientes, a través del paquete estadístico SPSS (versión 21). 


\section{Resultados}

En primer lugar, con el objetivo de conocer posibles diferencias en las puntuaciones medias del OMI-E entre sujetos que han mantenido contacto o recibido formación sobre las personas con trastorno mental, se llevan a cabo comparaciones de medias ( $t$ de Student) entre estudiantes de Educación Social y de otras titulaciones y entre sujetos que han mantenido o no un contacto previo (Tabla 1).

Tabla 1. Descriptivos y comparación de medias de los factores de la escala OMI-E, en función de las variable titulación y contacto previo

\begin{tabular}{|c|c|c|c|c|c|c|c|c|c|c|}
\hline OMI-E & Titulación & M & DT & $\mathrm{t}$ & $p$ & Contacto & $M$ & $D T$ & $t$ & $p$ \\
\hline \multirow{2}{*}{ Separatismo } & E Social & 21.88 & 4.56 & \multirow{2}{*}{-7.976} & \multirow{2}{*}{.000} & Sí & 22.78 & 5.22 & \multirow{2}{*}{-3.199} & \multirow{2}{*}{.001} \\
\hline & Otras & 25.55 & 5.15 & & & No & 24.36 & 4.94 & & \\
\hline \multirow{2}{*}{ Estereotipos } & E Social & 9.18 & 2.89 & \multirow{2}{*}{-3.946} & \multirow{2}{*}{.000} & Sí & 9.08 & 2.86 & \multirow{2}{*}{-4.828} & \multirow{2}{*}{.000} \\
\hline & Otras & 10.21 & 2.63 & & & No & 10.33 & 2.66 & & \\
\hline \multirow{2}{*}{ Restricción } & E Social & 7.20 & 2.54 & \multirow{2}{*}{-4.914} & \multirow{2}{*}{.000} & Sí & 7.48 & 2.55 & \multirow{2}{*}{-1.795} & \multirow{2}{*}{.073} \\
\hline & Otras & 8.32 & 2.29 & & & No & 7.90 & 2.40 & & \\
\hline \multirow{2}{*}{ Benevolencia } & E Social & 16.76 & 2.88 & \multirow{2}{*}{-4.688} & \multirow{2}{*}{.000} & Sí & 16.95 & 2.99 & \multirow{2}{*}{-2.859} & \multirow{2}{*}{.004} \\
\hline & Otras & 18.02 & 2.77 & & & No & 17.74 & 2.72 & & \\
\hline \multirow{2}{*}{$\begin{array}{l}\text { Predicción pe- } \\
\text { simista }\end{array}$} & E Social & 13.22 & 3.04 & \multirow{2}{*}{-1.381} & \multirow{2}{*}{.168} & Sí & 13.44 & 2.94 & \multirow{2}{*}{.432} & \multirow{2}{*}{.666} \\
\hline & Otras & 13.60 & 2.71 & & & No & 13.32 & 2.89 & & \\
\hline \multirow{2}{*}{ Estigmatización } & E Social & 8.25 & 2.30 & \multirow{2}{*}{-2.713} & \multirow{2}{*}{.007} & Sí & 8.53 & 2.39 & \multirow{2}{*}{.388} & \multirow{2}{*}{.698} \\
\hline & Otras & 8.83 & 2.17 & & & No & 8.45 & 2.09 & & \\
\hline \multirow{2}{*}{ Total } & E Social & 76.28 & 10.84 & \multirow{2}{*}{-7.790} & \multirow{2}{*}{.000} & Sí & 78.19 & 12.16 & \multirow{2}{*}{3.517} & \multirow{2}{*}{.000} \\
\hline & Otras & 84.87 & 11.17 & & & No & 82.31 & 10.80 & & \\
\hline
\end{tabular}

En cuanto a la comparación en función de la titulación cursada, los estudiantes de Educación Social, obtienen puntuaciones significativamente menores en los factores separatismo, estereotipos, restricción, benevolencia, estigmatización y en la puntuación total de la escala OMI-E, que aquellos estudiantes de otras titulaciones (Tabla 1). Igualmente, los resultados muestran que los sujetos que han tenido contacto con personas con trastorno mental, obtienen puntuaciones significativamente menores en los factores separatismo, estereotipos, benevolencia y en la puntuación total de la escala OMI-E que aquellos que no han tenido contacto con personas con trastorno mental (Tabla 1). 
Además, en la tabla 2, se presentan los resultados de las comparaciones intragrupos entre Educación Social y otras titulaciones en función si han mantenido o no contacto con personas con trastorno mental. Los resultados indican que los estudiantes de Educación Social que han mantenido contacto con personas con trastorno mental obtienen puntuación $(p \leq .05)$ menores en los factores separatismo, estereotipos, benevolencia y en la puntuación total de la escala OMI-E (Tabla 2). En cuanto a los estudiantes de otras titulaciones, no se han encontrado diferencias $(p \leq .05)$ en ninguna de las variables analizadas (Tabla 2 ).

Tabla 2. Descriptivos y comparación de medias de los factores de la escala OMI-E, en función de la variable contacto previo

\begin{tabular}{|c|c|c|c|c|c|c|c|c|c|}
\hline & \multirow{2}{*}{$\begin{array}{c}\text { Contacto } \\
\text { previo }\end{array}$} & \multicolumn{4}{|c|}{ E-Social } & \multicolumn{4}{|c|}{ Otras titulaciones } \\
\hline & & $M$ & $D T$ & $t$ & $p$ & $M$ & $D T$ & $t$ & $p$ \\
\hline \multirow{2}{*}{ Separatismo } & Sí & 21.07 & 4.32 & \multirow{2}{*}{-3.489} & \multirow{2}{*}{.001} & 25.18 & 5.43 & \multirow{2}{*}{-1.106} & \multirow{2}{*}{.270} \\
\hline & No & 23.06 & 4.67 & & & 26.03 & 4.79 & & \\
\hline \multirow{2}{*}{ Estereotipos } & Sí & 8.54 & 2.83 & \multirow{2}{*}{-4.574} & \multirow{2}{*}{.000} & 9.90 & 2.70 & \multirow{2}{*}{-1.919} & \multirow{2}{*}{.057} \\
\hline & No & 10.11 & 2.75 & & & 10.63 & 2.52 & & \\
\hline \multirow{2}{*}{ Restricción } & Sí & 7.03 & 2.50 & \multirow{2}{*}{-1.362} & \multirow{2}{*}{.174} & 8.15 & 2.47 & \multirow{2}{*}{-1.038} & \multirow{2}{*}{.301} \\
\hline & $\mathrm{No}$ & 7.46 & 2.57 & & & 8.49 & 2.00 & & \\
\hline \multirow{2}{*}{ Benevolencia } & Sí & 16.38 & 2.91 & \multirow{2}{*}{-2.643} & \multirow{2}{*}{.009} & 17.82 & 2.91 & \multirow{2}{*}{-1.159} & \multirow{2}{*}{.248} \\
\hline & No & 17.32 & 2.75 & & & 18.29 & 2.58 & & \\
\hline \multirow{2}{*}{ PredicciónPesimista } & Sí & 13.20 & 3.06 & \multirow{2}{*}{-.221} & \multirow{2}{*}{.825} & 13.80 & 2.71 & \multirow{2}{*}{1.052} & \multirow{2}{*}{.294} \\
\hline & No & 13.28 & 3.01 & & & 13.38 & 2.72 & & \\
\hline \multirow{2}{*}{ Estigmatización } & Sí & 8.28 & 2.44 & \multirow{2}{*}{.214} & \multirow{2}{*}{.831} & 8.91 & 2.26 & \multirow{2}{*}{.494} & \multirow{2}{*}{.622} \\
\hline & $\mathrm{No}$ & 8.22 & 2.09 & & & 8.75 & 2.04 & & \\
\hline \multirow{2}{*}{ Total } & $\overline{\text { Sí }}$ & 74.02 & 10.35 & \multirow{2}{*}{-3.975} & \multirow{2}{*}{.000} & 84.04 & 12.1 & & \\
\hline & No & 79.56 & 10.78 & & & 86.05 & 9.69 & -1.158 & .248 \\
\hline
\end{tabular}

\section{Discusión}

A través de la presente investigación se ha pretendido analizar si la formación recibida y el contacto previo sobre las personas con trastorno mental, son factores relevantes en las opiniones que tienen los estudiantes universitarios sobre dichas personas.

En cuanto a las diferencias en la opinión de los estudiantes universitarios en función de la formación recibida sobre las personas con trastorno mental, los resultados obtenidos señalan que aquellos que han recibido esa formación presentan mejores opiniones sobre ellas y, además, están dispuestos a mantener un mayor grado de contacto y vínculo con las mismas, poseen una imagen menos estereotipada, apuestan por una menor limitación de sus derechos, 
son más compasivos y no consideran el trastorno mental como algo que tiene que ser ocultado o un motivo de vergüenza. Estos resultados son similares a los obtenidos por Smith (2008), con una muestra de 188 participantes (pertenecientes a cuatro grupos: profesionales y estudiantes del ámbito de la salud mental, como trabajadores sociales y psicólogos, y otros pertenecientes a otros ámbitos), el cual halló que el entrenamiento en salud mental ejercía un efecto positivo -incluso mayor que el del contacto- sobre las actitudes estigmatizantes hacia las personas con trastorno mental, o por Pingani et al. (2016), con 311 estudiantes del ámbito de la salud, el cual considera que la formación recibida durante la carrera contribuye a que se mantengan menos estereotipos y comportamientos estigmatizantes; y, por último, Sharp et al. (2006), con una muestra de 213 estudiantes de Psicología que recibió una breve intervención educativa, los cuales apreciaron una disminución en la percepción de estas personas como peligrosas y en pensar que deben ser controladas, aunque, en su estudio, los efectos sobre la restricción social no se mantuvieron un mes después.

Sin embargo, en el presente estudio la predicción pesimista es la única subescala en la que la formación no parece tener un impacto significativo. En este sentido, Zellmann et al. (2014), encuentran que la opinión de los estudiantes de Trabajo Social no mejoraba en función de que éstos se encontrasen en un curso superior y que, incluso, mantenían una predicción más pesimista sobre los resultados de las intervenciones con estas personas. De acuerdo con estos mismos autores, dichos resultados pueden ser atribuidos a que, posiblemente, no se haya proporcionado información que pueda suponer un cambio significativo en las bases y actitudes previas que los estudiantes podrían mantener antes de comenzar sus estudios.

Un ejemplo de estas bases previas podría consistir en la creencia de atribuir la presencia de un trastorno mental, principalmente, a causas biogenéticas. Esto puede derivar en percibir a los sujetos con trastorno mental como impredecibles, antisociales y no responsables de sus acciones, así como en una mayor estigmatización (Mannarini y Boffo, 2013) hacia los mismos. Así, el hecho de que este tipo de creencias en torno a los trastornos mentales tuviese una incidencia significativa en la muestra, podría producir que en ésta se abogase por una mayor restricción de sus derechos sociales y que, además, al asociar la presencia del trastorno a factores biogenéticos, más estáticos, no se creyese en su recuperación.

Por otra parte, otro de los objetivos de la investigación se dirigía a analizar si el contacto previo con las personas con trastorno mental produce efectos significativos sobre la 
opinión de los estudiantes universitarios. Al respecto, los resultados muestran que aquellos sujetos que han mantenido dicho contacto mantienen una mejor opinión sobre las mismas. Asimismo, están dispuestos a establecer distancias sociales menores, poseen una imagen menos estereotipada y son más benevolentes respecto a éstas. Estos resultados reflejan los hallados en las investigaciones realizadas por Brown (2012), con 605 estudiantes de cursos introductorios de Psicología, el cual encontró una menor estigmatización entre quienes habían desarrollado ese contacto, o con los hallados por Nguyen et al. (2012), con una muestra de 280 estudiantes de Farmacia, los cuales señalan una reducción del estigma a través del contacto directo. En esta misma línea, Medina-Ortíz et al. (2010) hallaron una reducción en los miedos hacia ese colectivo y un mayor deseo de trabajar con el mismo, entre una muestra de 93 estudiantes de último curso de Medicina, después de que estos hubiesen realizado prácticas en una unidad de hospitalización.

En cambio, en el presente estudio, los resultados señalan que el hecho de que un sujeto establezca un contacto previo con las personas con trastorno mental no tiene por qué relacionarse con que éste considere necesario establecer menos restricciones en referencia a los derechos de esas personas, tenga mayores expectativas sobre su mejora y piense que la sociedad también las tenga o que crea que estos trastornos no son causa de vergüenza ni deban ocultarse. Al respecto, posiblemente se puede ver reflejada la relación entre estigmatización y la restricción de derechos y de la participación social de las personas que la sufren (Chen et al., 2016).

A su vez, el hecho de que el contacto previo no produjese cambios significativos en estas escalas puede estar en relación con las características del mismo. De esta manera, puede ocurrir que no se produjese con la suficiente intensidad o en unas condiciones que, aunque no sean necesarias (Pettigrew y Tropp, 2006), se relacionan positivamente con una reducción del prejuicio (Allport, 1958). Esto se encuentra reflejado en investigaciones como las realizadas por Fresán et al. (2012) y Omori et al. (2012), en las cuales el contacto se desarrolló en un contexto clínico, con ciertas nociones de verticalidad en la relación profesional-paciente y en el que los sujetos se podían encontrar en una fase clínica aguda.

Además, de manera similar a lo que ocurría en la formación, otra posible explicación puede relacionarse con que el contacto establecido no proporcionase información suficiente o 
significativa para modificar creencias como la atribución de la presencia del trastorno mental principalmente a partir de causas biogenéticas, con las posibles consecuencias que de esto se derivan, como asociar a estas personas con nociones como la impredecibilidad y la antisocialidad (Mannarini y Boffo, 2013).

Sin embargo, en lo que puede ser una de las principales aportaciones del presente estudio, si se profundiza un poco más en los resultados, se aprecia que los impactos producidos por el contacto previo sólo son significativos sobre aquellos sujetos que se encontraban cursando el Grado en Educación Social, es decir, en quienes también han recibido formación en la materia. Por ello, a su vez, los resultados parecen apuntar a que el contacto previo no produce diferencias significativas en la opinión de aquellos estudiantes que no han recibido dicha formación.

Estos resultados coinciden parcialmente con los encontrados por Eack y Newhill (2008), con 118 estudiantes de posgrado de Trabajo Social, y Eack et al. (2012), con 60 estudiantes de grado de esa misma disciplina. Estos autores sostenían que la formación y el contacto previo, por separado, no influyen positivamente en un cambio actitudinal entre los estudiantes de esa titulación hacia las personas con esquizofrenia. En cambio destacan su complementariedad: la formación puede favorecer que se establezcan contactos positivos y significativos. Además, destacan la relevancia de poseer conocimientos previos para que un contacto social cercano no derive en una interacción negativa. Al respecto, podría resaltarse la importancia de desarrollar acciones de educación basada en el contacto (Chen et al., 2016).

De otra parte, estos resultados pueden relacionarse con las teorías concernientes a los procesos cognitivos individuales de cambio que se producen a la hora de desconfirmar un estereotipo cuando se presenta nueva información (Crocker y Weber, 1983). En este sentido, el hecho de que los sujetos hayan recibido una formación previa puede contribuir a que posean un mayor volumen de información y, por ello, favorecer procesos asociados con la acumulación. Ésta consiste en una modificación gradual del estereotipo a través de una acumulación de información desconfirmatoria, donde importa la cantidad de la misma y la presencia una amplia muestra de sujetos que lo contradigan. Por el contrario, el hecho de no poseer ese volumen de información previa puede favorecer el que se produzcan procesos relacionados con los subtipos, ya que los contactos que se establezcan pueden no resultar significativos 
para desconfirmar el estereotipo y que, por ello, se creen subcategorías dentro del mismo en las que se va clasificando a los sujetos que lo desconfirman.

Asimismo, los resultados parecen apuntar a que, como expusieron Pettigrew y Tropp (2008), el conocimiento (factor cognitivo) ejerce, como mediador en la reducción del prejuicio a través del contacto, aunque cabe destacar que estos autores primaban el papel de la empatía y la reducción de la ansiedad durante el mismo (en este caso no se puede establecer la importancia de cada uno de ellos en este proceso, al desconocerse cómo se produjeron las diferentes interacciones, por lo que habría que profundizar sobre su rol al respecto).

\section{Conclusiones}

La formación recibida parece producir una mayor influencia que el contacto previo en la mejora de las opiniones que los estudiantes universitarios mantienen sobre las personas con trastorno mental. En este sentido, los resultados obtenidos muestran que mientras que el contacto previo se relaciona con diferencias significativas en las escalas de separatismo, estereotipos, benevolencia y en el cuestionario en general, la formación recibida parece relacionarse también con una mejora significativa en las subescalas de restricción y estigmatización, además de en las anteriormente citadas.

Por otra parte, la que puede ser la principal contribución de la investigación se basa en que el contacto previo únicamente parece provocar un cambio significativo en la opinión de los estudiantes universitarios si se establece cuando éstos han recibido o están recibiendo esa formación, en este caso, en materia de salud mental y reducción del estigma. De esta relación se desprende la importancia de proporcionar información previa a los estudiantes para que las interacciones que establezcan con las personas con trastorno mental resulten positivas y enriquecedoras de cara a mejorar sus actitudes y opiniones hacia estos y, en última instancia, a reducir una posible estigmatización.

No obstante, hay que mostrar cierta cautela en torno a los resultados obtenidos. Si bien estos parecen señalar la influencia de las dos variables (contacto previo y formación), las diferencias apreciadas en la opinión pueden deberse a otros factores que no han sido controlados en la presente investigación. Por ejemplo, se desconoce el nivel de incidencia en la muestra de diferentes bases previas como pueden ser las creencias sobre el origen de estos trastornos, 
como la atribución a factores biogenéticos (Mannarini y Boffo, 2013), de la formación, del contacto que habían experimentado con anterioridad, del sexo o de la motivación que llevó a los estudiantes a cursar una determinada titulación. En esta línea, Martínez et al. (2015), respecto a dicha motivación, señalan que existía un mayor interés por el altruismo (asociado con los afectos, sentimientos y valores personales del individuo) como motivo de elección de la carrera en titulaciones de corte más humanista y relacionadas con mentalidades de servicio a la comunidad, como es el caso de la Medicina, el Magisterio o la Psicología. Se desconoce si esto también puede ocurrir en titulaciones como Educación Social y si esa motivación previa hacia la ayuda y el servicio puede ejercer una influencia sobre la opinión que estos estudiantes mantienen sobre las personas con trastorno mental.

En función de lo anterior, se estima conveniente señalar que la presente investigación está sujeta a algunas limitaciones como no haber realizado una adaptación cultural del instrumento, en la línea de lo propuesto por Berra et al. (2009), la cual, por ejemplo, tuviese en cuenta procesos como las evaluaciones de los ítems; no haber tenido en cuenta algunas bases previas (Mannarini y Boffo, 2013; Martínez et al., 2015); disponer de una muestra que no estaba equilibrada, con un número mucho más elevado de mujeres que de hombres; o, por último, no haber diferenciado entre los distintos tipos de contacto - como los propuestos por Senra-Rivera et al. (2008) - o las condiciones en las que éste se desarrollaba.

En este sentido, resultaría muy interesante realizar futuras investigaciones en las que dichas limitaciones fuesen solventadas, mejorando la adaptación cultural del cuestionario, controlando esas bases (creencias, actitudes, etc.) previas, equilibrando la muestra o incluyendo los diferentes tipos de contacto que pueden establecerse y estudiando las condiciones del mismo. Además, podría resultar de especial interés realizar un estudio longitudinal en el que se analizase el impacto de la formación recibida a lo largo del Grado en Educación Social sobre los mismos sujetos, desde que éstos lo comenzasen hasta que lo finalizasen, así como incluir una medición de las causas - biogenéticas o psicosociales- a las que creen que se asocia el desarrollo de los trastornos mentales.

Por todo ello, se considera que la presente investigación ha proporcionado información útil, principalmente, de cara a mejorar la formación de los profesionales del ámbito de la salud mental y la rehabilitación psicosocial, como es el caso de los educadores sociales. A su vez, estos resultados pueden resultar de utilidad en la realización de campañas de reducción del 
estigma. En este sentido, se recomienda, por un lado, la utilización de estrategias basadas en la educación y la formación y, por otro, las acciones de educación basada en el contacto que combinen la formación con la interacción con personas con trastorno mental, ya que ambas pueden resultar eficaces en la lucha contra la estigmatización. En cambio, no se recomienda realizar campañas que impliquen un contacto con estas personas a menos que se haya recibido una formación previa, ya que el mismo puede resultar negativo o no tener una entidad suficiente para llegar a cambiar la opinión de quien lo establece.

\section{Referencias}

Álvarez, L. Y. y Pernía, L. C. (2007). Actitudes hacia el trastorno mental y hacia la búsqueda de ayuda psicológica profesional. Una revisión sistemática de estudios en Europa, Estados Unidos, Latinoamérica y el Caribe. MedUNAB, 10, 50-56.

Allport, G. W. (1958). The Nature of Prejudice. New York: Doubleday.

American Psychological Association. (2013). DSM 5. Washington: American Psychiatric Publishing.

Berra, S., Bustingorry, V., Henze, C., Díaz, M. P., Rajmil, L. y Butino, M. (2009). Adaptación transcultural del cuestionario KIDSCREEN para medir calidad de vida relacionada con la salud en población argentina de 8 a 18 años. Archivos argentinos de pediatría, 107, 307-314.

Boyd, J. E., Katz, E. P., Link, B. G. y Phelan, J. C. (2010). The relationship of multiple aspects of stigma and personal contact with someone hospitalized for mental illness, in a nationally representative sample. Social Psychiatry and Psychiatric Epidemiology, 45, 1063-1070. doi: 10.1007/s00127-009-0147-9

Brown, S. A. (2012). The Contribution of Previous Contact and Personality Traits to Severe Mental Illness Stigma. American Journal of Psychiatric Rehabilitation, 15, 274-289. doi: 10.1080/15487768.2012.703553

Chen, S. P., Koller, M., Krupa, T. y Stuart, H. (2016). Contact in the Classroom: Developing a Program Model for Youth Mental Health Contact-Based Anti-stigma Education. Community Mental Health Journal, 52, 281-293. doi: 10.1007/s10597-015-9944-7.

Cohen, J. y Struening, E. (1962). Opinions about mental illness in the personnel of two large mental hospitals. Journal of Abnormal Psychology, 64, 349-360. doi: $\underline{10.1037 / \mathrm{h} 0045526}$ 
Covarrubias, I. y Han, M. (2011). Mental Health Stigma about Serious Mental Illness among MSW Students: Social Contact and Attitude. Social Work, 56(4), 317-325. doi: $10.1093 / \mathrm{sw} / 56.4 .317$

Crocker, J. y Weber, R. (1983). Cognitive structure and stereotype change. En R. P. Bagozzi y A. M. Tybout (Eds.). Advances in Consumer Research (pp 459-463). Ann Abor: Association for Consumer Research.

Delgado, A. (2015). Estrategias psicosociales para el cambio de actitud hacia personas discriminadas por su discapacidad o trastorno mental. Revista Española de Discapacidad, 3(2), 27-39. doi: /10.5569/2340-5104.03.02.02

Eack, S. M. y Newhill, C. E. (2008). An Investigation of the Relations between Student Knowledge, Personal Contact, and Attitudes toward Individuals with Schizophrenia. Journal of Social Work Education, 44(3), 77-95. doi: 10.5175/JSWE.2008.200700009

Eack, S. M., Watson, A. C. y Newhill. C. E. (2012). Effects of severe mental illness education on MSW student attitudes about schizophrenia. Journal of Social Work Education, 48(3), 425-438. doi: 10.5175/JSWE.2012.201000042

Fernández, A. (2005). Multiculturalidad en contextos educativos y de desarrollo: relevancia de variables psicosociales. Electronic Journal of Research in Educational Psychology 3(1), 181-204

Fresán, A., Robles, R., Cota, M., Berlanga, C., Lozano, D. y Tena, A. (2012). Actitudes de mujeres estudiantes de psicología hacia las personas con esquizofrenia: relación con la percepción de agresividad y peligrosidad. Salud Mental, 35, 215-223.

Goffman, E. (2006). Estigma. La identidad deteriorada. Buenos Aires-Madrid: Amorrortu Editores. (Primera edición: 1963).

González, J. M., Tinsley, H. E. A. y Kreuder, K. R. (2002). Effects of Psychoeducational Interventions on Opinions of Mental Illness, Attitudes Toward Help Seeking, and Expectations About Psychotherapy in College Students. Journal of College Student Development, 43(1), 51-63.

Lolich, M. y Leiderman, E. A. (2008). Estigmatización en la esquizofrenia y otros trastornos mentales. VERTEX Revista Argentina de Psiquiatría, 19, 165-173.

Mannarini, S. y Boffo, M. (2013). Assessing Mental Disorder Causal Beliefs: A Latent Dimension Identification. Community Mental Health Journal, 49, 686-693. doi: $10.1007 / \mathrm{s} 10597-012-9581-3$ 
Martínez, A., Castro, M., Lucena, M. y Zurita, F. (2015). Elección de titulación universitaria y expectativas de resultados de los adolescentes de Granada. Revista Española de Orientación y Psicopedagogía, 26(3), 63-77.

Medina-Ortíz, O. et al. (2010). Medical students' attitudes towards mental disorders and their correlation with academic performance. Médicas UIS. Revista de los estudiantes de medicina de la Universidad Industrial de Santander, 23, 181-185.

Nguyen, E., Chen, T. F. y O'Reilly, C. L. (2012). Evaluating the impact of direct and indirect contact on the mental health stigma of pharmacy students. Social Psychiatry and Psychiatric Epidemiology, 47, 1087-1098. doi: 10.1007/s00127-011-0413-5

Nordt, C., Rössler, W. y Lauber, C. (2006). Attitudes of Mental Health Professionals Toward People With Schizophrenia and Major Depression. Schizophrenia Bulletin, 32(4), 709-714. doi: 10.1093/schbul/sbj065

Omori, A. et al. (2012). Influence of contact with schizophrenia on implicit attitudes towards schizophrenia patients held by clinical residents. BMC Psychiatry, 12(205), 1-8. doi: 10.1186/1471-244X-12-205

Pettigrew, T. F. y Tropp, L. R. (2006). A Meta-Analytic Test of Intergroup Contact Theory. Journal of Personality and Social Psychology, 90(5), 751-783. doi: 10.1037/00223514.90.5.751

Pettigrew, T. F. y Tropp, L. R. (2008). How does intergroup contact reduce prejudice? Metaanalytic tests of three mediators. European Journal of Social Psychology, 38, 922934. doi: 10.1002/ejsp.504

Pingani, L., Catellani, S., Del Vecchio, V., Sampogna, G., Ellefson, S. E., Rigatelli, M., ... Corrigan, P. W. (2016). Stigma in the context of schools: analysis of the phenomenon of stigma in a population of university students. BMC Psychiatry, 16(29), 1-7. dOI: $10.1186 / \mathrm{s} 12888-016-0734-8$

Senra-Rivera, C., Arriba-Rossetto, A. y Seoane-Pesqueira, G. (2008). Papel de la experiencia en la aceptación vs. rechazo del paciente con esquizofrenia. Revista Latinoamericana de Psicología, 40, 73-83.

Serrani, D. (2012). Construcción y validación de escala para evaluar las actitudes hacia los pacientes con enfermedad de Alzheimer y trastornos relacionados. Revista Española de Geriatría y Gerontología, 47(1), 7-14. doi: 10.1016/j.regg.2011.09.005

Sharp, W., Hargrove, D. S., Johnson, L. y Deal, W. P. (2006). Mental Health Education: An evaluation of a Classroom Based Strategy to Modify Help Seeking form Mental 
Health Problems. Journal of College Student Development, 47(4), 419-438. doi:10.1353/csd.2006.0051

Smith, A. L. (2008). An Investigation of Attitudes Towards Adults with Mental Illness Among Mental Health Professionals In-training, Non Mental Health Professionals Intraining, Mental Health Professionals, and Non Mental Health Professionals. (Tesis de Doctorado). University of North Carolina. Greensboro.

Universidad de Extremadura (2016). Grado en Educación Social. Recuperado de: http://www.unex.es/conoce-la-uex/centros/profesorado/titulaciones/info/salidas profe$\underline{\text { sionales?id }=0715}$

Zellmann, K. T., Madden, E. E. y Aguiniga, D. M. (2014). Bachelor of Social Work Students and Mental Health Stigma: Understanding Student Attitudes. Journal of Social Work Education, 50, 660-677. doi: 10.1080/10437797.2014.947900 